\title{
Double Prompt $J / \psi$ Hadroproduction in the Parton Reggeization Approach with High-Energy Resummation
}

\author{
Zhi-Guo He, ${ }^{1}$ Bernd A. Kniehl, ${ }^{1}$ Maxim A. Nefedov, ${ }^{1,2}$ and Vladimir A. Saleev ${ }^{2}$ \\ ${ }^{1}$ II. Institut für Theoretische Physik, Universität Hamburg, Luruper Chaussee 149, 22761 Hamburg, Germany \\ ${ }^{2}$ Samara National Research University, Moskovskoe Shosse 34, 443086 Samara, Russia
}

(Received 20 June 2019; published 17 October 2019)

\begin{abstract}
We study double prompt $J / \psi$ hadroproduction within the nonrelativistic-QCD factorization formalism adopting the parton Reggeization approach to treat initial-state radiation in a gauge invariant and infraredsafe way. We present first predictions for the cross section distributions in the transverse momenta of the subleading $J / \psi$ meson and the $J / \psi$ pair. Already at leading order in $\alpha_{s}$, these predictions as well as those for the total cross section and its distributions in the invariant mass $m_{\psi \psi \psi}$ and the rapidity separation $|Y|$ of the $J / \psi$ pair nicely agree with recent ATLAS and CMS Collaboration measurements, except for the large$m_{\psi л \psi}$ and large- $|Y|$ regions, where the predictions substantially undershoot the data. In the latter regions, Balitsky-Fadin-Kuraev-Lipatov resummation is shown to enhance the cross sections by up to a factor of 2 and so to improve the description of the data.
\end{abstract}

DOI: 10.1103/PhysRevLett.123.162002

Despite concerted experimental and theoretical endeavors ever since the discovery of the $J / \psi$ meson more than four decades ago, its production mechanism has remained mysterious; for a recent review, see Ref. [1]. The factorization approach [2] to nonrelativistic QCD (NRQCD) [3] endowed with velocity scaling rules [4] for the longdistance matrix elements (LDMEs), which is by far the most acceptable candidate theory for heavy-quarkonium production and decay and has been elaborated at next-toleading order (NLO), has been challenged by the longstanding $J / \psi$ polarization puzzle [5] and by the inadequate description of $\eta_{c}$ hadroproduction data with $J / \psi$ LDMEs converted via heavy-quark spin symmetry [6]. The high flux of incoming partons at the LHC allows us to study $J / \psi$ production more thoroughly, also in association with other charmonia, bottomonia, $W$ or $Z$ bosons, so as to pin down the $J / \psi$ production mechanism. Among these production processes, double $J / \psi$ hadroproduction is of special interest because $J / \psi$ formation takes place there twice, making this particularly sensitive to the nonperturbative aspects of NRQCD [7]. Moreover, this is believed to be an exquisite laboratory to study double parton scattering (DPS) and to extract its key parameter, the effective cross section $\sigma_{\text {eff }}$ [8].

In recent years, double prompt $J / \psi$ hadroproduction has been measured extensively by the LHCb [9], CMS [10],

Published by the American Physical Society under the terms of the Creative Commons Attribution 4.0 International license. Further distribution of this work must maintain attribution to the author(s) and the published article's title, journal citation, and DOI. Funded by SCOAP . and ATLAS [11] Collaborations at the CERN LHC, and by the D0 Collaboration [12] at the FNAL Tevatron. On the theoretical side, the complete NRQCD results at leading order (LO) have been obtained recently [13]. For some channels, the relativistic corrections [14] and NLO QCD corrections [15] are also available. The available NRQCD predictions can explain the $\mathrm{LHCb}$ and D0 data, and to some extent also the CMS data, reasonably well. However, these single-parton scattering (SPS) predictions only amount to a few percent of the CMS data in the regions of large invariant mass $m_{\psi \psi \psi}$ or rapidity separation $|Y|=\left|y_{1}-y_{2}\right|$ of the $J / \psi$ pair, although the color-octet contributions, in particular those involving $t$ channel gluon exchanges, enhance the QCD-corrected [15] color-singlet contribution of $g g \rightarrow 2 c \bar{c}\left({ }^{3} S_{1}^{[1]}\right)$ there by more than 1 order of magnitude. The value of $\sigma_{\text {eff }}$ extracted by fitting the DPS contribution on top of this [12] is considerably smaller than typical values from other processes, and the resulting SPS plus DPS results still undershoot the CMS data in the upper $m_{\psi \psi}$ and $|Y|$ bins [16].

As noticed in Ref. [13], the CMS kinematic conditions [10] render $2 \rightarrow 3$ subprocesses predominant, which enter at NLO in the collinear parton model (CPM). However, a complete NLO NRQCD computation is presently out of reach from the technical point of view. On the conceptual side, the conventional NRQCD factorization formalism needs to be extended to cope with the double $P$-wave case [17]. Moreover, the perturbative expansion is spoiled for small values of the $J / \psi$ pair transverse momentum $p_{T}^{\psi \psi \psi}$. The characteristic scale $\mu \sim\left[\left(4 m_{c}\right)^{2}+\left(p_{T}^{\psi / \mu}\right)^{2}\right]^{1 / 2}$ of the hard-scattering processes of double $J / \psi$ hadroproduction satisfies $\Lambda_{\mathrm{QCD}} \ll \mu \ll \sqrt{S}$, where $\Lambda_{\mathrm{QCD}}$ is the asymptotic 
scale parameter and $\sqrt{S}$ is the center-of-mass energy. We are thus accessing the high-energy Regge regime, where the NLO QCD corrections can be largely accounted for through the unintegrated parton distribution functions (UPDFs) in the parton Reggeization approach (PRA) [18] based on Lipatov's effective field theory formulated with the non-Abelian gauge invariant action [19]. The PRA has already been successfully applied to the interpretation of measurements of single heavy-quarkonium hadroproduction [20,21]. In the large- $|Y|$ region, the two $J / \psi$ mesons are well separated, obeying multi-Regge kinematics (MRK). For subprocesses containing $t$ channel gluon exchange type diagrams, there will be large logarithms of the form $\left(\alpha_{s} \ln |s / t|\right)^{n}$ in the higher-order QCD corrections, where $s$ and $t$ are the Mandelstam variables of the partonic $2 \rightarrow 2$ Born process. Such large logarithms can be resummed by the Balitsky-Fadin-Kuraev-Lipatov (BFKL) formalism [22]. Recently, BFKL resummation has been studied for single $J / \psi$ [23] and $J / \psi$ plus jet [24] inclusive hadroproduction. In this Letter, we will take a crucial step towards a full-fledged NLO NRQCD study of double prompt $J / \psi$ hadroproduction, by adopting the PRA and performing BFKL resummation.

Owing to the PRA and NRQCD factorization, the cross section of inclusive double prompt $J / \psi$ hadroproduction can be expressed as

$$
\begin{aligned}
d \sigma^{\mathrm{PRA}}(A B \rightarrow 2 J / \psi+X)= & \sum_{m, n, H_{1}, H_{2}} \int \frac{d x_{1}}{x_{1}} \int \frac{d^{2} \mathbf{k}_{1 T}}{\pi} \int \frac{d x_{2}}{x_{2}} \int \frac{d^{2} \mathbf{k}_{2 T}}{\pi} \Phi_{R^{+} / A}\left(x_{1}, t_{1}, \mu^{2}\right) \Phi_{R^{-} / B}\left(x_{2}, t_{2}, \mu^{2}\right) \\
& \times d \hat{\sigma}_{m n}^{\mathrm{PRA}}\left\langle\overline{\mathcal{O}}^{H_{1}}(m)\right\rangle\left\langle\overline{\mathcal{O}}^{H_{2}}(n)\right\rangle,
\end{aligned}
$$

where $d \hat{\sigma}_{m n}^{\mathrm{PRA}}$ is the short-distance coefficient (SDC) of the partonic subprocess $R^{+}\left(k_{1}\right) R^{-}\left(k_{2}\right) \rightarrow c \bar{c}(m) c \bar{c}(n)+X$, $\Phi_{R^{ \pm} / A, B}\left(x_{1,2}, t_{1,2}, \mu^{2}\right)$ are the UPDFs of the Reggeized gluons $R^{ \pm}$with four-momenta $k_{1,2}^{\mu}=x_{1,2} K_{1,2}^{\mu}+k_{1,2 T}^{\mu}$ and virtualities $t_{1,2}=-k_{1,2}^{2}=\mathbf{k}_{1,2 T}^{2}, K_{1,2}^{\mu}$ are the fourmomenta of the colliding hadrons $A, B$ with light-cone components $\quad K_{1}^{-}=K_{2}^{+}=0 \quad\left(K_{i}^{ \pm}=K_{i}^{0} \pm K_{i}^{3}\right), \quad$ and $\left\langle\overline{\mathcal{O}}^{H}(m)\right\rangle$ is the product of LDME $\left\langle\mathcal{O}^{H}(m)\right\rangle$ of $H=$ $J / \psi, \chi_{c J}, \psi^{\prime}$ and the branching ratio $\operatorname{BR}(H \rightarrow J / \psi+X)$, with the understanding that $\operatorname{BR}(H \rightarrow J / \psi+X)=1$ if $H=J / \psi$. Since partonic subprocesses initiated by Reggeized quarks and antiquarks are greatly suppressed by their UPDFs, we may disregard them here. Furthermore, we may neglect the $\chi_{c 0}$ feed-down contribution because $\operatorname{BR}\left(\chi_{c 0} \rightarrow J / \psi+X\right)=1.40 \%$ [25] is so small.

Representative Feynman diagrams for the partonic subprocess $R^{+} R^{-} \rightarrow c \bar{c}(m) c \bar{c}(n)$ at $\mathrm{LO}$ in $\alpha_{s}$ are depicted in Figs. 1(a) and 1(b). By the Feynman rules of Ref. [19], they come in the same topologies as those for $g g$ fusion in the $\mathrm{CPM}$. To discuss the BFKL resummation effect conveniently, we divide the partonic subprocesses into three categories, according to the order in $\alpha_{s}$ where $t$ channel gluon exchanges emerge for the first time, namely, (i) LO $t$ channel (LT), with $m, n={ }^{1} S_{0}^{[8]},{ }^{3} S_{1}^{[8]},{ }^{3} P_{J}^{[1,8]}$; (ii) NLO $t$

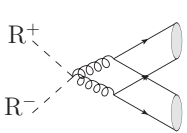

(a)

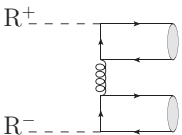

(b)

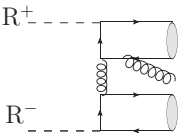

(c)

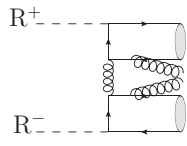

(d)
FIG. 1. Typical Feynman diagrams for $R^{+} R^{-} \rightarrow c \bar{c}(m) c \bar{c}(n)$ of types (a) non- $t$ channel gluon exchange and $t$ channel gluon exchange at (b) LO, (c) NLO, and (d) NNLO in $\alpha_{s}$. channel (NLT), with $m={ }^{3} S_{1}^{[1]}$ and $n={ }^{1} S_{0}^{[8]},{ }^{3} S_{1}^{[8]},{ }^{3} P_{J}^{[1,8]}$; and (iii) NNLO $t$ channel (NNLT), with $m, n={ }^{3} S_{1}^{[1]}$; see Figs. 1(b)-1(d).

We first compute the LO contributions to all the three categories. Because of lack of space, we relegate the details of our calculation to a separate paper. In contrast to other $k_{T}$ factorization approaches [26], the PRA yields gauge invariant SDCs with off-shell initial-state partons, which provides a strong check for our analytic calculations. In the collinear limits $t_{1,2} \rightarrow 0$, we recover the CPM formulas [13], which constitutes yet another nontrivial check. In the numerical analysis, we adopt the Kimber-Martin-Ryskin scheme [27] to generate the UPDFs from the LO CPM PDFs of Ref. [28], which come with $\alpha_{s}\left(M_{Z}^{2}\right)=0.13939$. We choose the renormalization and factorization scales to be $\mu_{r}=\mu_{f}=\xi\left[\left(4 m_{c}\right)^{2}+\bar{p}_{T}^{2}\right]^{1 / 2}$, where $m_{c}=1.5 \mathrm{GeV}$, $\bar{p}_{T}=\left(p_{T}^{H_{1}}+p_{T}^{H_{2}}\right) / 2$, and $\xi$ is varied between $1 / 2$ and 2 about its default value 1 to estimate the scale uncertainty. In the case of feed down from $H=\chi_{c_{1}}, \chi_{c_{2}}, \psi^{\prime}$, we put $p_{T}^{J / \psi}=p_{T}^{H} M_{J / \psi} / M_{H}$, which is a good approximation because $p_{T}^{J / \psi} \gg M_{H}-M_{J / \psi}$ [29]. For the $J / \psi, \chi_{c J}$, and $\psi^{\prime}$ LDMEs, we use the values specified in Table I. The color-singlet results have been derived from the Buchmüller-Tye potential in Ref. [30]. The color-octet results have been fitted to LHC data of inclusive single charmonium hadroproduction [31] in the very theoretical framework described above; they supersede pre-LHC results [20]. For $H=J / \psi, \psi^{\prime}$, there is a strong correlation between $\left\langle\mathcal{O}^{H}\left({ }^{1} S_{0}^{[8]}\right)\right\rangle$ and $\left\langle\mathcal{O}^{H}\left({ }^{3} P_{0}^{[8]}\right)\right\rangle$, so that only a linear combination of them can be determined. We may thus put $\left\langle\mathcal{O}^{H}\left({ }^{3} P_{0}^{[8]}\right)\right\rangle=0$. We have checked that the theoretical uncertainties in our predictions for double prompt $J / \psi$ 
TABLE I. Adopted values of LO NRQCD LDMEs in units of $\mathrm{GeV}^{3}$.

\begin{tabular}{llllllll}
\hline \hline$\left\langle\mathcal{O}^{J / \psi}\left({ }^{3} S_{1}^{[1]}\right)\right\rangle\left\langle\mathcal{O}^{J / \psi}\left({ }^{1} S_{0}^{[8]}\right)\right\rangle\left\langle\mathcal{O}^{J / \psi}\left({ }^{3} S_{1}^{[8]}\right)\right\rangle$ & $\left\langle\mathcal{O}^{\psi^{\prime}}\left({ }^{3} S_{1}^{[1]}\right)\right\rangle$ & $\left\langle\mathcal{O}^{\psi^{\prime}}\left({ }^{1} S_{0}^{[8]}\right)\right\rangle$ & $\left\langle\mathcal{O}^{\psi^{\prime}}\left({ }^{3} S_{1}^{[8]}\right)\right\rangle$ & $\left\langle\mathcal{O}^{\chi_{c 0}}\left({ }^{3} P_{0}^{[1]}\right)\right\rangle / m_{c}^{2}$ & $\left\langle\mathcal{O}^{\chi_{c 0}}\left({ }^{3} S_{1}^{[8]}\right)\right\rangle\left\langle\mathcal{O}^{J / \psi(}\left(\psi^{\prime}\right)\right.$ & $\left.\left({ }^{3} P_{J}^{[8]}\right)\right\rangle / m_{c}^{2}$ \\
\hline \hline
\end{tabular}

hadroproduction due to this freedom are negligible. All other input parameters are adopted from Ref. [25].

The CMS data of prompt double $J / \psi$ hadroproduction were taken at $\sqrt{S}=7 \mathrm{TeV}$ requiring for each $J / \psi$ meson to be in the rapidity range $|y|<2.2$ and to satisfy a $y$ dependent minimum- $p_{T}$ cut, as described in Eq. (3.3) of Ref. [10]. The CMS total cross section $\sigma_{\mathrm{CMS}}=(1.49 \pm$ $0.07 \pm 0.13) \mathrm{nb}$ agrees with our LO NRQCD prediction $\sigma_{\mathrm{CMS}}^{\mathrm{PRA}}=1.68_{-0.78}^{+1.32} \mathrm{nb}$ within errors. The CMS $p_{T}^{\psi \psi}, m_{\psi \psi}$, and $|Y|$ distributions are compared with our predictions in Figs. 2(a)-2(c), respectively. There is generally very good agreement, except for the upper two $m_{\psi \psi}$ bins and the upmost $|Y|$ bin, where the predictions undershoot the data. The advancement of the PRA beyond the CPM is most striking for Fig. 2(a) because $p_{T}^{\psi \psi \psi}=0$ at LO in the latter case, but it is also significant for the $m_{\psi \psi}$ and $|Y|$ distributions, as may be observed by comparing Figs. 2(b) and 2(c) with their CPM counterparts in Figs. 3 and 4 of Ref. [13]. In both cases, the predictions are substantially increased in the first three bins, so as to nicely match the data, while the $K$ factors are of order unity in the upmost bins.

The UPDF uncertainty may be assessed from Fig. 2(a), which also shows the evaluations using the set produced from our default PDF set [28] as described in Ref. [32] and the set of Ref. [33]. The latter rapidly falls off with increasing $p_{T}^{\psi \psi}$ and significantly undershoots the data in the upper $p_{T}^{\psi \psi}$ bins. This is in line with Ref. [34], where the UPDFs of Ref. [33] were found to yield a poor description of LHCb data of single prompt $J / \psi$ production [35] at large $p_{T}^{\psi}$. This opens a novel perspective to constrain UPDFs. To estimate the LDME uncertainty, we repeat the unresummed LO PRA evaluation in the upmost $|Y|$ bin of Fig. 2(c), which is most sensitive to the color-octet LDMEs, using in turn the NLO CPM sets of Refs. [36,37], albeit this is slightly inconsistent. Since Ref. [36] does not provide $\chi_{c J}$ and $\psi^{\prime}$ LDMEs, we use those of Ref. [37] also here. We thus find an enhancement by $25 \%$ and a reduction by $7 \%$ with respect to our default result, respectively. For a more detailed LDME analysis, see Ref. [38].

ATLAS took their data at $\sqrt{S}=8 \mathrm{TeV}$ imposing the acceptance cuts $p_{T}>8.5 \mathrm{GeV}$ and $|y|<2.1$ on each $J / \psi$ meson [11]. They separately studied the central (I) and forward (II) $y$ regions of the subleading $J / \psi$ meson $\left(J / \psi_{2}\right)$, with $p_{2 T}<p_{1 T}$, namely, $\left|y_{2}\right|<1.05$ and $1.05<\left|y_{2}\right|<2.1$. Their respective total cross sections $\sigma_{\text {ATLAS,I }}=(82.2 \pm$ $8.3 \pm 6.3) \mathrm{pb}$ and $\sigma_{\mathrm{ATLAS}, \mathrm{II}}=(78.3 \pm 9.2 \pm 6.6) \mathrm{pb}$ are both compatible with our LO PRA predictions $\sigma_{\text {ATLAS,I }}^{\text {PRA }}=$ $133.6_{-52.2}^{+89.6} \mathrm{pb}$ and $\sigma_{\mathrm{ATLAS}, \mathrm{II}}^{\mathrm{PRA}}=105.2_{-41.6}^{+73.8} \mathrm{pb}$. Their respective $p_{2 T}, p_{T}^{\psi \psi}$, and $m_{\psi \mu \psi}$ distributions are compared with our LO PRA predictions in Fig. 3. We find fairly good agreement for the $p_{2 T}$ and $p_{T}^{\psi \psi}$ distributions, especially in region II, with regard to both normalization and line shape. In particular, the predictions faithfully reproduce the peaks of the measured $p_{T}^{\psi \psi}$ distributions. As for the $m_{\psi \psi \psi}$ distributions, there is decent agreement for $m_{\psi \mu \psi} \lesssim 40 \mathrm{GeV}$, while the predictions significantly undershoot the data in the upper $m_{\psi \mu \psi}$ bins, as in the CMS case above.

Also the LHCb [9] and D0 [12] Collaboration measurements reasonably agree with our PRA predictions. The LHCb [9] data at $\sqrt{S}=7 \mathrm{TeV}$, with acceptance cuts $p_{T}<$ $10 \mathrm{GeV}$ and $2<y<4.5$ on each $J / \psi$ meson, yield $\chi^{2} /$ d.o.f. $=12.8 / 5$ with respect to our LO PRA prediction with $\xi=1$ in the perturbatively safe region $m_{\psi \mu}>9 \mathrm{GeV}$ [13], with experimental and theoretical errors overlapping.
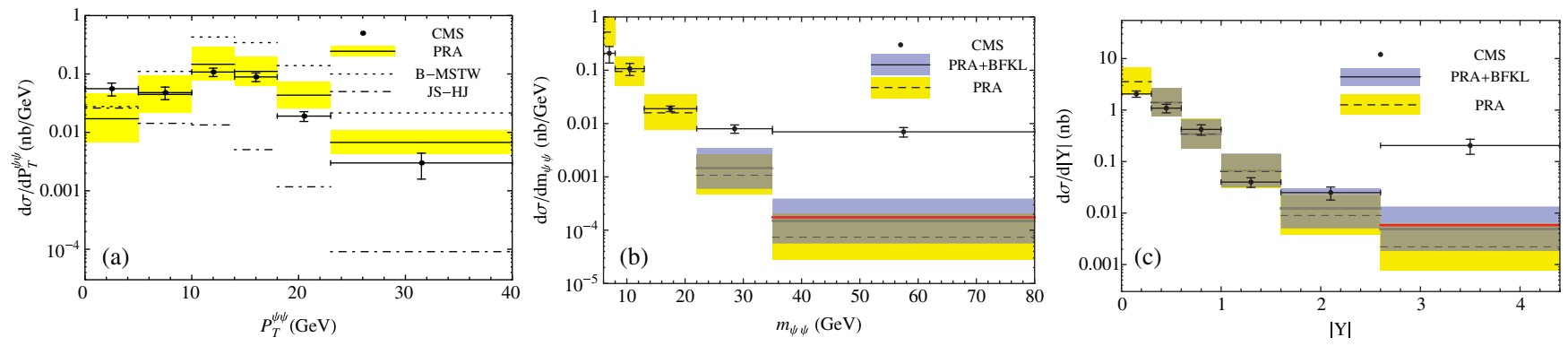

FIG. 2. The (a) $p_{T}^{\psi \psi}$, (b) $m_{\psi \psi}$, and (c) $|Y|$ distributions of double prompt $J / \psi$ production measured by the CMS Collaboration [10] are compared to our LO NRQCD predictions in the PRA without (dashed lines) and with BFKL resummation (solid lines) including their scale uncertainties (yellow and blue bands). Adding the total NLO* NLT contributions on top of the central LO NRQCD predictions in the PRA with BFKL resummation yields the red solid lines. Frame (a) also contains the evaluations with the UPDF sets of Refs. [28,32] (B-MSTW, dotted lines) and Ref. [33] (JS-HJ, dot-dashed lines). 

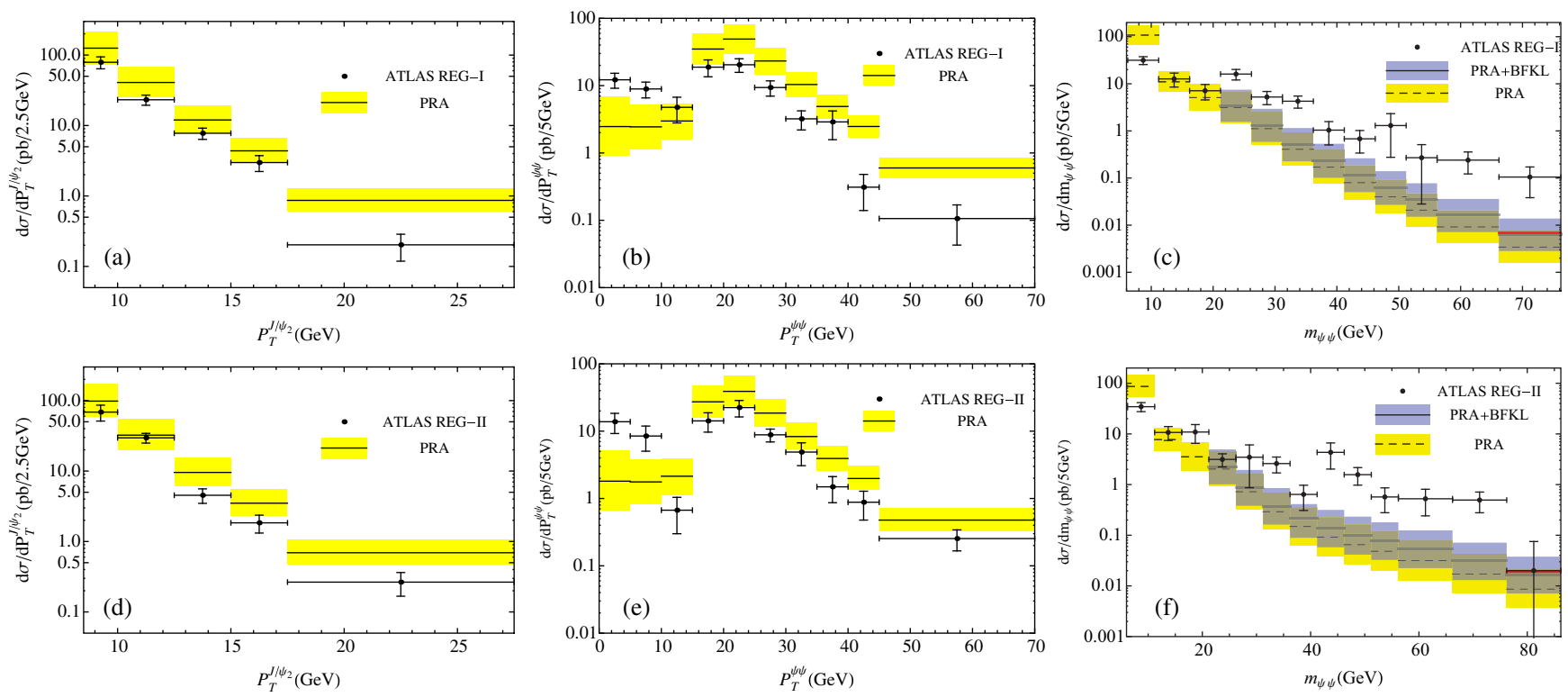

FIG. 3. As in Fig. 2, but for the $p_{2 T}$ (left column), $p_{T}^{\psi \psi}$ (middle column), and $m_{\psi \mu \psi}$ (right column) distributions measured by the ATLAS Collaboration [11] in regions I (upper row) and II (lower row).

The fiducial cross section $\sigma_{\text {SPS }}=70 \pm 6$ (stat) \pm 22 (syst) fb determined by the D0 Collaboration [12] at $\sqrt{S}=7 \mathrm{TeV}$, with cuts $p_{T}<10 \mathrm{GeV}$ and $2<y<4.5$, nicely agrees with our central LO PRA prediction $\sigma_{\mathrm{SPS}}^{\mathrm{PRA}}=81.1 \mathrm{fb}$, where we have included the reduction factor due to the acceptance cuts on the decay muons [12] determined in Ref. [39].

Although the LO CPM relationship $m_{\psi \psi}=2\left[\left(2 m_{c}\right)^{2}+\right.$ $\left.\left(p_{T}^{J / \psi}\right)^{2}\right]^{1 / 2} \cosh (|Y| / 2)$ [13] is evaded by the PRA, detailed analysis of the $m_{\psi \psi \psi}$ and $|Y|$ distributions reveals that they are strongly correlated in the large- $m_{\psi \psi}$ and $-|Y|$ regions. As expected, the LT contributions greatly dominate there. Specifically, they make up about $90 \%$ or more of the total PRA predictions in the upmost $m_{\psi \psi}$ bin in Fig. 2(b), the upmost $|Y|$ bin in Fig. 2(c), the upmost $m_{\psi \psi \psi}$ bin in Fig. 3(c), and the upper four $m_{\psi \mu}$ bins in Fig. 3(f). $t$ channel gluon exchanges, which appear in the LT contributions already at LO, generate large logarithmic corrections of the type $\left(\alpha_{s} \ln |s / t|\right)^{n}$ in higher orders, which can be efficiently included via BFKL resummation.

BFKL resummation in the leading-logarithmic (LL) approximation is implemented by replacing the SDCs in Eq. (1) with

$$
\begin{aligned}
& \frac{d \hat{\sigma}_{m n}^{\mathrm{BFKL}}}{d \mathbf{p}_{1 T} d y_{1} d \mathbf{p}_{2 T} d y_{2}} \\
& =\int \frac{d^{2} \mathbf{q}_{T} d^{2} \mathbf{q}_{T}^{\prime}}{(4 \pi)^{2} S^{2} x_{1} x_{2}} \\
& \quad \times \Psi_{+}^{(m)}\left(\mathbf{k}_{1 T}, \mathbf{p}_{1 T}, y_{1}\right) G\left(\mathbf{q}_{T}, \mathbf{q}_{T}^{\prime}, Y\right) \Psi_{-}^{(n)}\left(\mathbf{k}_{2 T}, \mathbf{p}_{2 T}, y_{2}\right),
\end{aligned}
$$

where $\Psi_{+}^{(m)}\left(\mathbf{k}_{1 T}, \mathbf{p}_{1 T}, y_{1}\right)$ and $\Psi_{-}^{(n)}\left(\mathbf{k}_{2 T}, \mathbf{p}_{2 T}, y_{2}\right)$ are the impact factors describing the partonic subprocesses $R^{+}\left(\mathbf{k}_{1 T}\right) R^{-}\left(\mathbf{q}_{T}\right) \rightarrow[c \bar{c}(m)]\left(\mathbf{p}_{1 T}\right)$ and $R^{-}\left(\mathbf{k}_{2 T}\right) R^{+}\left(-\mathbf{q}_{T}^{\prime}\right) \rightarrow$ $[c \bar{c}(n)]\left(\mathbf{p}_{2 T}\right)$, obtained from the appropriate $2 \rightarrow 1$ PRA matrix elements in Ref. [20] as explained in Ref. [40], and $G\left(\mathbf{q}_{T}, \mathbf{q}_{T}^{\prime}, Y\right)$ is the BFKL Green function given by Eq. (3.80) in Ref. [40], generated from the initial condition $G\left(\mathbf{q}_{T}, \mathbf{q}_{T}^{\prime}, 0\right)=\delta^{(2)}\left(\mathbf{q}_{T}-\mathbf{q}_{T}^{\prime}\right)$ via LL BFKL evolution in $Y$ [22]; see Fig. 4 for a schematic representation of Eq. (2). The resulting hadronic cross section is denoted as $d \sigma^{\mathrm{BFKL}}$.

$G\left(\mathbf{q}_{T}, \mathbf{q}_{T}^{\prime}, Y\right)$ depends exponentially on $\alpha_{s}\left(\mu^{2}\right)$, which may produce a potentially large theoretical uncertainty. Several approaches have been proposed to remedy this. As frequently done [41], we adopt here the one [42] based on a non-Abelian physical renormalization scheme choice in connection with the Brodsky-Lepage-Mackenzie optimal scale setting [43]. There remains a reference scale $\mu_{0}$. We choose $\mu_{0}=\xi\left[\left|\mathbf{k}_{1 T}\right|\left|\mathbf{k}_{2 T}\right|\right]^{1 / 2}$ and vary $\xi$ from $1 / 2$ to 2

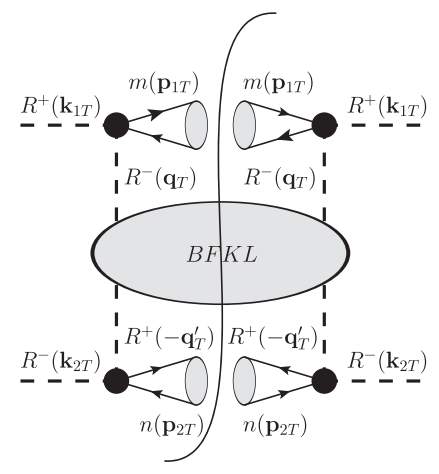

FIG. 4. Schematic representation of Eq. (2). 
about its default value 1 to estimate the residual scale uncertainty in $G\left(\mathbf{q}_{T}, \mathbf{q}_{T}^{\prime}, Y\right)$.

We merge the full LO PRA calculation $d \sigma^{\mathrm{PRA}}$, appropriate in the small- $|Y|$ region, and the LL-resummed LT contribution $d \sigma^{\mathrm{BFKL}}$, appropriate in the large- $|Y|$ region, as

$$
d \sigma^{\mathrm{PRA}+\mathrm{BFKL}}=d \sigma^{\mathrm{PRA}}+d \sigma^{\mathrm{BFKL}}-d \sigma^{\mathrm{BFKL}, 0},
$$

where the asymptotic term $d \sigma^{\mathrm{BFKL}, 0}$, which is obtained from $d \sigma^{\text {BFKL }}$ by replacing $G\left(\mathbf{q}_{T}, \mathbf{q}_{T}^{\prime}, Y\right)$ with $G\left(\mathbf{q}_{T}, \mathbf{q}_{T}^{\prime}, 0\right)$, is to avoid double counting. Equation (3) smoothly interpolates from $d \sigma^{\mathrm{PRA}}$ at small $|Y|$ values to $d \sigma^{\mathrm{BFKL}}$ at large $|Y|$ values.

The BFKL-improved PRA predictions thus evaluated are also included in Figs. 2 and 3. Their uncertainties are obtained by combining the PRA and BFKL ones in quadrature. They exceed the PRA uncertainties only moderately, which indicates that the scale uncertainties in $G\left(\mathbf{q}_{T}, \mathbf{q}_{T}^{\prime}, Y\right)$ are well under control. In the $p_{2 T}$ and $p_{T}^{\psi / \mu}$ distributions and in the lowest few bins of the $m_{\psi \mu \psi}$ and $|Y|$ distributions, the BFKL resummation effects are so insignificant that we refrain from displaying the BFKLimproved results. On the other hand, these effects are significant in the upper $m_{\psi \psi \psi}$ and $|Y|$ bins, where they may even double the pure PRA results, so as to reduce the shortfall with respect to the CMS [10] and ATLAS [11] data. In the latter case, even agreement is reached in some medium $m_{\psi \mu \nu}$ bins. However, large gaps remain in the upmost $|Y|$ bin of CMS and the upmost few $m_{\psi \psi}$ bins of CMS and ATLAS, to be explained by DPS. Contrary to naïve expectations, the optimal scale turns out to be larger than $\mu_{0}$, so that using the latter instead leads to an enhancement of the BFKL-improved results, by factors of 1.0,1.0,1.1, 1.5, and 4.1 in the second to sixth $|Y|$ bins of CMS.

Quantitative extractions of DPS contributions from the remaining discrepancies are likely to be meaningful only after the complete NLO NRQCD corrections are available, for the following reasons. First, conventional NRQCD factorization is known to break down at NLO for double $P$-wave channels [17]. The quantitative influence of this is presently unclear. Second, the NLO NRQCD corrections to the NLT subprocesses can be quite sizable because their $\mathcal{O}\left(\alpha_{s}\right)$ suppression is expected to be compensated by the relatively large values of the color-singlet LDMEs $\left\langle\mathcal{O}^{J / \psi}\left({ }^{3} S_{1}^{[1]}\right)\right\rangle$ and $\left\langle\mathcal{O}^{\psi^{\prime}}\left({ }^{3} S_{1}^{[1]}\right)\right\rangle$. For the ${ }^{3} S_{1}^{[1]}+{ }^{1} S_{0}^{[8]} /{ }^{3} P_{1,2}^{[1]}$ channels, the type of diagrams in Fig. 1(c) form a gauge invariant subset, but not for the ${ }^{3} S_{1}^{[1]}+{ }^{3} S_{1}^{[8]}$ channel because of $g \rightarrow c \bar{c}\left({ }^{3} S_{1}^{[8]}\right)$ formation. Their leading large- $|Y|$ contributions can be estimated via the gauge invariant MRKasymptotic formalism, already used to evaluate $d \sigma^{\mathrm{BFKL}, 0}$ for the LT subprocesses in Eq. (3). We have checked for the $R^{+} R^{-} \rightarrow c \bar{c}\left({ }^{3} S_{1}^{[1]}\right) c \bar{c}\left({ }^{1} S_{0}^{[8]}\right) g$ subprocess that our MRK approximation reproduces the exact result for the $t$ channel gluon exchange type diagrams in the upmost CMS $|Y|$ bin within a factor of 1.2.

In this way, we find that such partial NLO (NLO ${ }^{*}$ ) results for the individual $(m, n)$ channels among the NLT subprocesses can be up to 100 times larger than the LO PRA results for these channels in the upper $|Y|$ and $m_{\psi \psi \psi}$ bins. The effect of adding the total NLO* NLT contribution on top of the central LO NRQCD prediction in the PRA with BFKL resummation is shown for the upmost $m_{\psi \psi}$ and $|Y|$ bins in Figs. 2 and 3. In Fig. 2(c), this amounts to $45 \%$ and $16 \%$ for direct and prompt production, respectively. The total NLO* NLT contributions will, in turn, be enhanced by BFKL resummation, which we leave for future work.

To summarize, we have pushed the NRQCD factorization approach to double prompt $J / \psi$ hadroproduction beyond LO in two important ways. On the one hand, we have incorporated multiple gluon radiation off the initial state via the PRA, which, unlike other $k_{T}$ factorization approaches frequently used in the literature [26], ensures for the SDCs to be manifestly gauge invariant, infrared safe, and devoid of artificial kinematic cuts. On the other hand, we have resummed, via BFKL evolution in $|Y|$, the LLs of the form $\left(\alpha_{s} \ln |s / t|\right)^{n}$ arising from $t$ channel gluon exchanges in the LT subprocesses [see Fig. 1(b)], which would otherwise inevitably invalidate the fixed-order treatment at large $|Y|$ and $m_{\psi \psi}$ values. This consolidates the theoretical basis for meaningful extractions of the DPS key parameter $\sigma_{\text {eff }}$.

M. A. N. was supported by the Alexander von Humboldt Foundation through a Research Fellowship for Postdoctoral Researchers. V. A.S. was supported in part by Samara University Competitiveness Improvement Program under Tasks No. 3.5093.2017/8.9 and No. 0777-20170007. This work was supported in part by BMBF Grant No. 05H18GUE and DFG Grant No. KN365/12-1.

[1] N. Brambilla et al. (Quarkonium Working Group), Heavy quarkonium: progress, puzzles, and opportunities, Eur. Phys. J. C 71, 1534 (2011); QCD and strongly coupled gauge theories: challenges and perspective, Eur. Phys. J. C 74, 2981 (2014).

[2] G. T. Bodwin, E. Braaten, and G. P. Lepage, Rigorous QCD analysis of inclusive annihilation and production of heavy quarkonium, Phys. Rev. D 51, 1125 (1995); Erratum, Phys. Rev. D 55, 5853(E) (1997).

[3] W. E. Caswell and G. P. Lepage, Effective lagrangians for bound state problems in QED, QCD, and other field theories, Phys. Lett. 167B, 437 (1986).

[4] G. P. Lepage, L. Magnea, C. Nakhleh, U. Magnea, and K. Hornbostel, Improved nonrelativistic QCD for heavy-quark physics, Phys. Rev. D 46, 4052 (1992).

[5] M. Butenschoen and B. A. Kniehl, $J / \psi$ Polarization at the Tevatron and the LHC: Nonrelativistic-QCD Factorization at the Crossroads, Phys. Rev. Lett. 108, 172002 (2012); 
Next-to-leading order tests of non-relativistic-QCD factorization with $J / \psi$ yield and polarization, Mod. Phys. Lett. A 28, 1350027 (2013).

[6] M. Butenschoen, Z.-G. He, and B. A. Kniehl, $\eta_{c}$ Production at the LHC Challenges Nonrelativistic QCD Factorization, Phys. Rev. Lett. 114, 092004 (2015).

[7] V. Barger, S. Fleming, and R. J. N. Phillips, Double gluon fragmentation to $J / \psi$ pairs at the tevatron, Phys. Lett. B 371, 111 (1996).

[8] C. H. Kom, A. Kulesza, and W. J. Stirling, Pair Production of $J / \psi$ as a Probe of Double Parton Scattering at LHCb, Phys. Rev. Lett. 107, 082002 (2011).

[9] R. Aaij et al. (LHCb Collaboration), Observation of $J / \psi$ pair production in $p p$ collisions at $\sqrt{s}=7 \mathrm{TeV}$, Phys. Lett. B 707, 52 (2012); Measurement of the $J / \psi$ pair production cross-section in $p p$ collisions at $\sqrt{s}=13 \mathrm{TeV}$, J. High Energy Phys. 06 (2017) 047; Erratum, J. High Energy Phys. 10 (2017) 68(E).

[10] V. Khachatryan et al. (CMS Collaboration), Measurement of prompt $J / \psi$ pair production in $p p$ collisions at $\sqrt{s}=7$ TeV, J. High Energy Phys. 09 (2014) 094.

[11] M. Aaboud et al. (ATLAS Collaboration), Measurement of the prompt $J / \psi$ pair production cross-section in $p p$ collisions at $\sqrt{s}=8 \mathrm{TeV}$ with the ATLAS detector, Eur. Phys. J. C 77, 76 (2017).

[12] V. M. Abazov et al. (D0 Collaboration), Observation and studies of double $J / \psi$ production at the Tevatron, Phys. Rev. D 90, 111101(R) (2014).

[13] Z.-G. He and B. A. Kniehl, Complete Nonrelativistic-QCD Prediction for Prompt Double $J / \psi$ Hadroproduction, Phys. Rev. Lett. 115, 022002 (2015).

[14] Y.-J. Li, G.-Z. Xu, K.-Y. Liu, and Y.-J. Zhang, Relativistic correction to $J / \psi$ and $\Upsilon$ pair production, J. High Energy Phys. 07 (2013) 051.

[15] L.-P. Sun, H. Han, and K.-T. Chao, Impact of $J / \psi$ pair production at the LHC and predictions in nonrelativistic QCD, Phys. Rev. D 94, 074033 (2016).

[16] J.-P. Lansberg and H.-S. Shao, $J / \psi$-pair production at large momenta: Indications for double parton scatterings and large $\alpha_{s}^{5}$ contributions, Phys. Lett. B 751, 479 (2015).

[17] Z.-G. He, B. A. Kniehl, and X.-P. Wang, Breakdown of Nonrelativistic QCD Factorization in Processes Involving Two Quarkonia and its Cure, Phys. Rev. Lett. 121, 172001 (2018).

[18] B. A. Kniehl, M. A. Nefedov, and V. A. Saleev, Promptphoton plus jet associated photoproduction at HERA in the parton Reggeization approach, Phys. Rev. D 89, 114016 (2014); A. V. Karpishkov, M. A. Nefedov, and V. A. Saleev, $B \bar{B}$ angular correlations at the LHC in the parton Reggeization approach merged with higher-order matrix elements, ibid. 96, 096019 (2017).

[19] L. N. Lipatov, Gauge invariant effective action for high energy processes in QCD, Nucl. Phys. B452, 369 (1995).

[20] B. A. Kniehl, D. V. Vasin, and V. A. Saleev, Charmonium production at high energy in the $k_{T}$-factorization approach, Phys. Rev. D 73, 074022 (2006).

[21] B. A. Kniehl, V. A. Saleev, and D. V. Vasin, Bottomonium production in the Regge limit of QCD, Phys. Rev. D 74, 014024 (2006); V. A. Saleev, M. A. Nefedov, and A. V. Shipilova, Prompt $J / \psi$ production in the Regge limit of
QCD: from the Tevatron to the LHC, ibid. 85, 074013 (2012); M. A. Nefedov, V. A. Saleev, and A. V. Shipilova, Prompt $\Upsilon(n S)$ production at the LHC in the Regge limit of QCD, ibid. 88, 014003 (2013); B. A. Kniehl, M. A. Nefedov, and V. A. Saleev, $\psi(2 S)$ and $\Upsilon(3 S)$ hadroproduction in the parton Reggeization approach: Yield, polarization, and the role of fragmentation, ibid. 94, 054007 (2016).

[22] E. A. Kuraev, L. N. Lipatov, and V. S. Fadin, Multiregge processes in the Yang-Mills theory, Zh. Eksp. Teor. Fiz. 71, 840 (1976) [Sov. Phys. JETP 44, 443 (1976)]; I. I. Balitsky and L. N. Lipatov, The pomeranchuk singularity in quantum chromodynamics, Yad. Fiz. 28, 1597 (1978) [Sov. J. Nucl. Phys. 28, 822 (1978)].

[23] P. Kotko, L. Motyka, M. Sadzikowski, and A. M. Staśto, BFKL Pomeron loop contribution in diffractive photoproduction and inclusive hadroproduction of $J / \psi$ and $\Upsilon$, J. High Energy Phys. 07 (2019) 129.

[24] R. Boussarie, B. Ducloué, L. Szymanowski, and S. Wallon, Forward $J / \psi$ and very backward jet inclusive production at the LHC, Phys. Rev. D 97, 014008 (2018).

[25] M. Tanabashi et al. (Particle Data Group), Review of Particle Physics, Phys. Rev. D 98, 030001 (2018).

[26] S. P. Baranov and A.H. Rezaeian, Prompt double $J / \psi$ production in proton-proton collisions at the LHC, Phys. Rev. D 93, 114011 (2016).

[27] M. A. Kimber, A. D. Martin, and M. G. Ryskin, Unintegrated parton distributions, Phys. Rev. D 63, 114027 (2001).

[28] A. D. Martin, W. J. Stirling, R. S. Thorne, and G. Watt, Parton distributions for the LHC, Eur. Phys. J. C 63, 189 (2009).

[29] Y.-Q. Ma, K. Wang, and K.-T. Chao, QCD radiative corrections to $\chi_{c J}$ production at hadron colliders, Phys. Rev. D 83, 111503(R) (2011).

[30] E. J. Eichten and C. Quigg, Quarkonium wave functions at the origin, Phys. Rev. D 52, 1726 (1995).

[31] G. Aad et al. (ATLAS Collaboration), Measurement of $\chi_{c 1}$ and $\chi_{c 2}$ production with $\sqrt{s}=7 \mathrm{TeV} p p$ collisions at ATLAS, J. High Energy Phys. 07 (2014) 154; Measurement of the production cross-section of $\psi(2 \mathrm{~S}) \rightarrow J / \psi(\rightarrow$ $\left.\left.\mu^{+} \mu^{-}\right) \pi^{+} \pi^{-}\right)$in $p p$ collisions at $\sqrt{s}=7 \mathrm{TeV}$ at ATLAS, J. High Energy Phys. 09 (2014) 079; Measurement of the differential cross-sections of prompt and non-prompt production of $J / \psi$ and $\psi(2 \mathrm{~S})$ in $p p$ collisions at $\sqrt{s}=7$ and $8 \mathrm{TeV}$ with the ATLAS detector, Eur. Phys. J. C 76, 283 (2016); V. Khachatryan et al. (CMS Collaboration), Measurement of $J / \psi$ and $\psi(2 S)$ Prompt Double-Differential Cross Sections in $p p$ Collisions at $\sqrt{s}=7 \mathrm{TeV}$, Phys. Rev. Lett. 114, 191802 (2015).

[32] J. Blümlein, On the $k_{\perp}$ dependent gluon density of the proton, arXiv:hep-ph/9506403.

[33] H. Jung and G. P. Salam, Hadronic final state predictions from CCFM: the hadron-level Monte Carlo generator CAsCAde, Eur. Phys. J. C 19, 351 (2001); F. Hautmann and H. Jung, Transverse momentum dependent gluon density from DIS precision data, Nucl. Phys. B883, 1 (2014).

[34] R. Maciuła, A. Szczurek, and A. Cisek, $J / \psi$-meson production within improved color evaporation model with the $k_{T}$-factorization approach for $c \bar{c}$ production, Phys. Rev. D 99, 054014 (2019). 
[35] R. Aaij et al. (LHCb Collaboration), Measurement of $J / \psi$ production in $p p$ collisions at $\sqrt{s}=7 \mathrm{TeV}$, Eur. Phys. J. C 71, 1645 (2011).

[36] M. Butenschoen and B. A. Kniehl, World data of $J / \psi$ production consolidate nonrelativistic QCD factorization at next-to-leading order, Phys. Rev. D 84, 051501(R) (2011).

[37] B. Gong, L.-P. Wan, J.-X. Wang, and H.-F. Zhang, Polarization for Prompt $J / \psi$ and $\psi(2 s)$ Production at the Tevatron and LHC, Phys. Rev. Lett. 110, 042002 (2013).

[38] J.- P. Landsberg, H.-S. Shao, N. Yamanaka, and Y.-J. Zhang, Prompt $J / \psi$-pair production at the LHC: impact of loopinduced contributions and of the colour-octet mechanism, arXiv:1906.10049.

[39] C.-F. Qiao and L.-P. Sun, $J / \psi$ pair production at the Tevatron with $\sqrt{s}=1.96 \mathrm{TeV}$, Chin. Phys. C 37, 033105 (2013).
[40] Yu. V. Kovchegov and E. Levin, Quantum Chromodynamics at High Energy (Cambridge University Press, Cambridge, 2012).

[41] B. Ducloué, L. Szymanowski, and S. Wallon, Evidence for High Energy Resummation Effects in Mueller-Navelet Jets at the LHC, Phys. Rev. Lett. 112, 082003 (2014); F. Caporale, D. Yu. Ivanov, B. Murdaca, and A. Papa, Mueller-Navelet jets in next-to-leading order BFKL: theory versus experiment, Eur. Phys. J. C 74, 3084 (2014); Erratum, Eur. Phys. J. C 75, 535(E) (2015).

[42] S. J. Brodsky, V. S. Fadin, V. T. Kim, L. N. Lipatov, and G. B. Pivovarov, The QCD Pomeron with optimal renormalization, Pis'ma Zh. Éksp. Teor. Fiz. 70, 161 (1999) [JETP Lett. 70, 155 (1999)].

[43] S. J. Brodsky, G. P. Lepage, and P. B. Mackenzie, On the elimination of scale ambiguities in perturbative quantum chromodynamics, Phys. Rev. D 28, 228 (1983). 